\title{
Estudo biomecânico comparativo da resistência a forças de compressão entre os parafusos pediculares poliaxiais com travamento tipo Dytech ${ }^{\circledR}$ e parafusos pediculares poliaxiais com travamento tipo Lock $1^{\circledR}$
}

\author{
Biomechanical comparative study of compression strength between \\ poliaxial screws Dytech ${ }^{\circledR}$ Locked type and poliaxial screws Lock $1^{\circledR}$ \\ Estudio biomecánico comparativo de la resistencia a fuerzas de \\ compresión entre los tornillos pediculares poliaxiales con traba tipo \\ Dytech ${ }^{\circledR}$ y tornillos pediculares poliaxiales con traba tipo Lock $1^{\circledR}$
}

\author{
Mauricio Pagy de Calais Oliveira' \\ Luiz Cláudio de Moura França ${ }^{2}$ \\ Marcelo Gonçalves Ruggani ${ }^{3}$ \\ Luiz Olímpio Garcia Pedrosa ${ }^{4}$ \\ Bruno Pinto Coelho Fontes ${ }^{4}$ \\ Rodrigo D'Alessandro de Macedo ${ }^{4}$ \\ Rafael Gonçalves Duarte ${ }^{5}$ \\ Marcelo Gonçalves Pereira Duarte ${ }^{6}$
}

\section{RESUMO}

Objetivo: Comparar a rigidez de um sistema de fixação pedicular composto por parafusos pediculares poliaxiais de travamento tipo Dytech ${ }^{\circledR}$ com outro composto por parafusos pediculares poliaxiais com travamento do tipo Lock $1^{\circledR}$, submetidos a forças de compressão. Métodos: A amostra utilizada para avaliar os sistemas de fixação respeitou as regras do padrão formulado pela American Society for Testing Materials (ASTM) no ensaio F1717-04. Os modelos foram divididos em: Grupo 1, composto pelos ensaios de parafusos poliaxiais com sistema Dytech ${ }^{\circledR}$ de travamento, e o

\begin{abstract}
Objective: To compare rigidity of a pedicle fixation system composed of poliaxial pedicle screws with Dytech ${ }^{\circledR}$ lock system to one composed of poliaxial screws with Lock $1^{\circledR}$ system, submitted to compression strengths. Methods: The sample used to evaluate the fixation systems respected the norms of the standard described by the American Society for Testing Materials (ASTM) in trial F1717-04. The models were divided into: Group 1, composed of trials using poliaxial screws with Dytech $^{\circledR}$ system, and Group 2, used poliaxial screws with Lock $1^{\circledR}$ system.
\end{abstract}

\section{RESUMEN}

Objetivo: comparar la rigidez de un sistema de fijación pedicular compuesto por tornillos pediculares poliaxiales de traba tipo Dytech ${ }^{\circledR}$ con otro compuesto por tornillos pediculares poliaxiales con traba del tipo Lock $1^{\circledR}$, sometidos a fuerzas de compresión. Métodos: la muestra utilizada para evaluar los sistemas de fijación respetó las reglas del patrón formulado por la American Society for Testing Materials (ASTM) en el ensayo F1717-04. Los modelos fueron divididos en: Grupo 1, compuesto por los ensayos de tornillos poliaxiales con sistema Dytech ${ }^{\circledR}$ de \footnotetext{
versidade Federal de São Carlos UFSCar - São Carlos (SP), Brasil.

'Ortopedista e Cirurgião de Coluna dos Hospitais Vera Cruz e Socor - Belo Horizonte (MG), Brasil. 2Ortopedista e Cirurgião de Coluna dos Hospitais Mater Dei e Baleia - Belo Horizonte (MG), Brasil.

${ }^{3}$ Neurocirurgião e Cirurgião de Coluna do Hospital Vera Cruz - Belo Horizonte (MG), Brasil.

${ }^{4}$ Ortopedista e Cirurgião de Coluna do Hospital da Baleia - Belo Horizonte (MG), Brasil.

${ }^{5}$ Ortopedista do Hospital Mater Dei - Belo Horizonte (MG), Brasil.

${ }^{6}$ Ortopedista do Hospital Life Center - Belo Horizonte (MG), Brasil.

Conflito de interesses: Este trabalho teve apoio não-financeiro da indústria Baumer para a sua realização.
}

Trabalho realizado no Centro Mineiro de Cirurgia da Coluna - Belo Horizonte (MG), Brasil e no Centro de Caracterização e Desenvolvimento de Materiais da Uni- 
Grupo 2, formado por parafusos poliaxiais com travamento tipo Lock $1^{\circledR}$. Foram testados três conjuntos completos montados. Cada sistema foi testado uma única vez por ser esse um ensaio destrutivo. O instrumental implantado foi produzido com titânio de mesma origem. Os grupos experimentais foram submetidos a testes mecânicos na máquina universal de ensaios EMIC, modelo EMIC DL $10000^{\circledR}$. Resultados: Os resultados de compressão nas amostras do Grupo 1 tiveram uma carga máxima média de $967,17 \mathrm{~N}$ e carga de escoamento média de $804,71 \mathrm{~N}$. Nas amostras do Grupo 2 tivemos uma carga máxima média de 906,04 N e carga de escoamento média de 834,56 N. A respeito da integridade dos instrumentais metálicos usados, não foi observado nenhum tipo de escorregamento ou soltura de porcas, parafusos ou outros componentes. Conclusão: O sistema de parafusos poliaxiais com travamento tipo Dytech ${ }^{\circledR}$ apresentou valores de rigidez maiores, enquanto o sistema de parafusos com travamento tipo Lock $1{ }^{\circledR}$ mostrou deslocamento máximo maior.

DESCRITORES: Parafusos ósseos; Placas ósseas; Fixação interna de fratura/instrumentação; Titânio; Biomecânica; In vitro
Three complete sets were tested. Each system was tested only once because it's a destructive essay. The implanted components were produced with titanium coming from the same origin. The experimental groups were submitted to mechanical tests in a universal testing machine EMIC, model EMIC DL $10000^{\circledR}$. Results: The samples of Group 1 showed an average maximum charge of 967,17 $N$, and an average discharging load of 814,71 N. The samples of Group 2 showed an average maximum charge of 906,04 $\mathrm{N}$, and an average discharging load of 834,56 N. About the integrity of the used metallic components, was not noted any kind of failure in all of them. Conclusion: The pofilaxial screws system with Dytech $^{\circledR}$ lock has presented greater rigidity, while the Lock $1^{\circledR}$ system screws showed greater maximum displacement.

KEYWORDS: Bone screws; Bone plates; Fracture fixation, internal/instrumentation; Titanium; Biomechanics; In vitro traba, y el Grupo 2, formado por tornillos poliaxiales con traba tipo Lock $1^{\circledR}$. Fueron probados tres conjuntos completos montados. Cada sistema fue probado una vez por ser ese un ensayo destructivo. El instrumental implantado fue producido con titanio del mismo origen. Los grupos experimentales fueron sometidos a pruebas mecánicas en la máquina universal de ensayos EMIC, modelo EMIC DL $10000^{\circledR}$. Resultados: los resultados de compresión en las muestras del Grupo 1 tuvieron una carga máxima promedio de $967.17 \mathrm{~N}$ y carga de declive promedio de $804.71 \mathrm{~N}$. En las muestras del Grupo 2, tuvimos una carga máxima promedio de 906.04 $N$ y carga de declive promedio de 834.56 N. Con respecto a la integridad de los instrumentales metálicos usados, no fue observado ningún tipo de deslizamiento o soltura de tuercas, tornillos y otros componentes. Conclusión: el sistema de tornillos poliaxiales con traba tipo Dytech ${ }^{\mathbb{R}}$ presentó valores de rigidez mayores, mientras que el sistema de tornillos con traba tipo Lock $1^{\circledR}$ mostró un desplazamiento mayor.

DESCRIPTORES: Tornillos óseos; Placas óseas; Fijación interna de fracturas/instrumentación; Titanio; Biomecánica; In vitro

\section{INTRODUÇÃO}

A fixação da coluna vertebral por meio de parafusos pediculares é assunto amplamente discutido na literatura recente ${ }^{1-3}$. Autores como Boucher ${ }^{4}$ e, posteriormente, Roy Camile $^{5,6}$ sempre são lembrados no desenvolvimento desse tipo de fixação. A partir da década de 1980, notou-se um maior arsenal disponível para a fixação pedicular ${ }^{3}$. Foi observado também, a partir dessa época, o surgimento de novos conceitos biomecânicos referentes à fixação da coluna vertebral ${ }^{7}$.

Modificações nos modelos dos parafusos e na técnica cirúrgica ${ }^{8,9}$ têm sido implementadas com o objetivo de aumentar a estabilidade do sistema e minimizar suas complicações ${ }^{8,10,11}$. Em algumas situações, necessita-se de alguns dispositivos para melhor adaptação dos parafusos pediculares às hastes ${ }^{10}$. Os parafusos poliaxiais constituem importante recurso para isso, uma vez que apresentam maior flexibilidade de encaixe com as hastes. Isso tende a diminuir a quebra do material ${ }^{8}$.

Foi feita pesquisa em indexadores bibliográficos e não conseguimos encontrar na literatura um estudo biomecânico realizado in vitro dentro das normas ASTM F1717$04^{12}$, comparando sistemas diferentes de fixação poliaxial, montados em corpos de prova e submetidos a forças de compressão.

O objetivo deste trabalho foi comparar a rigidez a cargas de flexão de um sistema de fixação pedicular composto por parafusos pediculares poliaxiais com travamento tipo Dytech $^{\circledR}$ (porca e parafuso interno) e parafusos pediculares poliaxiais com travamento tipo Lock $1^{\circledR}$ (único). 


\section{MÉTODOS}

Foram utilizados dois sistemas diferentes de travamento dos parafusos poliaxiais associados com hastes. Esses sistemas foram montados e testados conforme o padrão descrito pela American Society for Testing Materials (ASTM), no ensaio F1717-04. A análise foi realizada por meio de um modelo de vertebrectomia com um largo espaço entre os blocos de polietileno, para avaliação mecânica dos implantes de coluna submetidos a diversas forças ${ }^{12}$. Os testes foram realizados no Centro de Caracterização e Desenvolvimento de Materiais da Universidade Federal de São Carlos - UFSCar. Os componentes metálicos implantáveis, tais como parafusos, porcas, hastes e conectores eram específicos para um sistema lombar e torácico. Os ensaios foram divididos em três estágios: A) confecção de blocos de polietileno de peso molecular ultra elevado (UHMWPE) (Figura 1); B) confecção do dispositivo para realização do ensaio; C) montagem do sistema como um todo, que consistiu em dois blocos de polietileno fixados com parafusos pediculares poliaxiais unidos por duas hastes longitudinais, além de um sistema de conexão transversal dentro das normas ASTM F 1717-04 (Figura 2). Cada sistema utilizou três kits montados, e cada kit foi testado uma única vez por ser um ensaio destrutivo. As amostras foram divididas em dois grupos, sendo o Grupo 1 composto pelos ensaios com parafusos poliaxiais com sistema de fixação através de porca e parafuso interno do tipo Dytech ${ }^{\circledR}$ e, no Grupo 2, fixação através de parafusos poliaxiais do tipo Lock $1^{\circledR}$. Nos dois grupos os parafusos apresentavam $6 \mathrm{~mm}$ de diâmetro e $45 \mathrm{~mm}$ de comprimento. A identificação das amostras, a especificação e a codificação dos componentes de cada sistema de fixação estão apresentados nas Tabelas $1 \mathrm{e} 2$.

As figuras 3 e 4 mostram os modelos de parafusos Dytech ${ }^{\circledR}$ e Lock $1{ }^{\circledR}$ respectivamente.

Os resultados obtidos foram comparados por meio de análise estatística, buscando-se identificar: o desvio de compensação de $2 \%$ de escoamento $(\mathrm{mm})$, o deslocamento elástico $(\mathrm{mm})$, a carga de escoamento na compressão $(\mathrm{N})$,
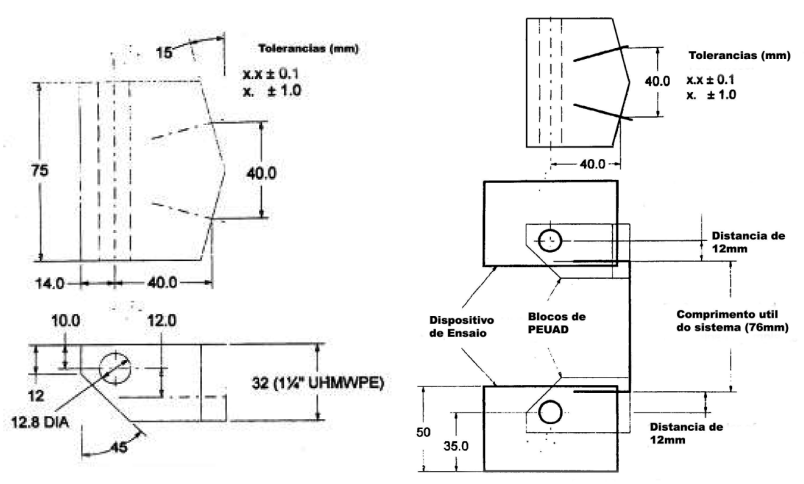

Figura 1

Medidas dos modelos dos blocos de polietileno.

Fonte: Universidade Federal de São Carlos. Essas figuras foram obtidas da norma americana ASTM F 1717. Trata-se, portanto, de um ensaio normatizado.

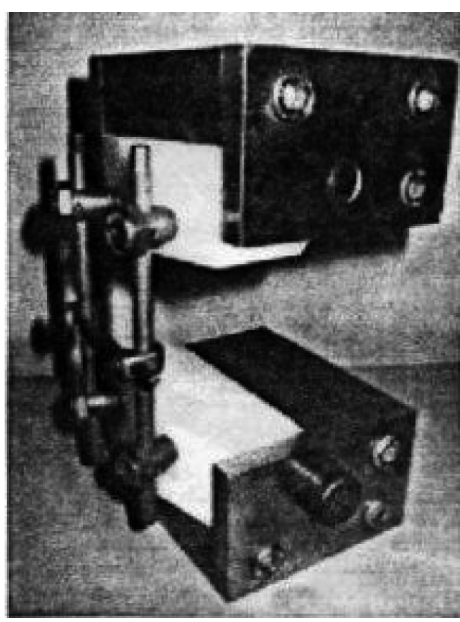

Figura 2

Figura do dispositivo padrão montado para os testes. Fonte: Universidade Federal de São Carlos. Essa montagem é exigida pela norma americana ASTM F 1717, ou seja, é um ensaio normatizado em que a montagem é determinada pela referida norma.

\section{TABELA 1 - Identificação das amostras e dos componentes metálicos implantáveis dos parafusos poliaxiais com travamento tipo Dytech $^{\circledR}$}

Grupo 1 Designação CCDM

Sistema de fixação de coluna

vertebral - Fixação pedicular Dytech ${ }^{\circledR}$

com fixação através de porca e

MET 050344

parafuso interno

Descrição do Produto

Código

Barra longitudinal

780.D. 120

Conector de Barras

792.A

Barra Transversal

771.A.75

Porca

766

Parafuso para coluna $6,0 \times 45 \mathrm{~mm}$

819.A.645

Parafuso interno

791.2

Fonte: Universidade Federal de São Carlos - UFSCar - São Carlos (SP), Brasil.

TABELA 2 - Identificação das amostras e dos componentes metálicos implantáveis dos parafusos poliaxiais com travamento tipo Lock $1^{\circledR}$

\begin{tabular}{lc}
\hline Grupo 2 & Designação CCDM \\
\hline Sistema de fixação de coluna & \\
vertebral - Fixação pedicular Lock & MET 06-000779 \\
Descrição do Produto & Código \\
Barra longitudinal & 879.120 \\
Barra transversal & 879. A.75 \\
Parafuso poliaxial & 877. B.640 \\
Parafuso trava & 877.1 \\
Conector & 879. B \\
Conector aberto & $879 . C$ \\
\hline
\end{tabular}

Fonte: Universidade Federal de São Carlos - UFSCar - São Carlos (SP), Brasil. 


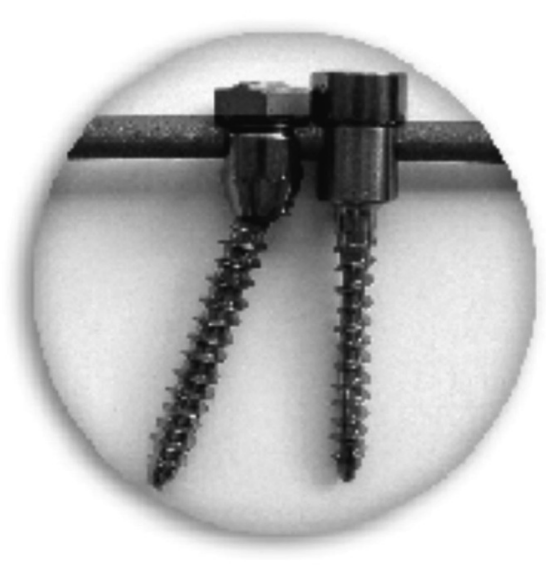

Figura 3

Figura do parafuso poliaxial Dytech.

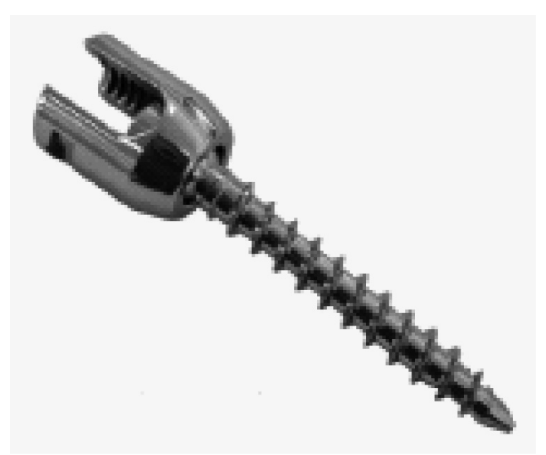

Figura 4

Figura do parafuso poliaxial Lock 1 .

a rigidez $(\mathrm{N} / \mathrm{mm})$, o deslocamento máximo na compressão $(\mathrm{mm})$ e a carga máxima de compressão $(\mathrm{N})$. Podemos encontrar a definição e ilustração desses termos na figura 5 .

Os componentes metálicos implantados referem-se ao titânio de mesma origem. Foram utilizados, para o ensaio de compressão, três corpos de prova para cada grupo, com implantes de Ti6AI4V ELI do Sistema de Fixação de Coluna Vertebral - Fixação Pedicular Dytech ${ }^{\circledR}$ - Baumer S.A. no Grupo 1 e implantes de Ti6AI4V ELI do Sistema de Fixação de Coluna Vertebral - Fixação Pedicular Lock 1 - Baumer S.A. no Grupo 2.

Os grupos experimentais foram submetidos a testes mecânicos na máquina universal de ensaios EMIC, modelo EMIC DL 10000, a uma velocidade de $5 \mathrm{~mm} / \mathrm{min}$, com a finalidade de avaliar a deformação do sistema quando submetido a forças de compressão, sendo que a velocidade máxima prescrita, por norma, é de $25 \mathrm{~mm} / \mathrm{min}$. Essa velocidade é normatizada pela norma americana ASTM F 1717.

Os dados foram analisados por meio do teste $t$ de Student, utilizando o programa SPSS 16.0.

\section{RESULTADOS}

Os resultados experimentais obtidos pelo ensaio de compressão dos Grupos 1 e 2 são apresentados nas tabelas 3 e 4 respectivamente.

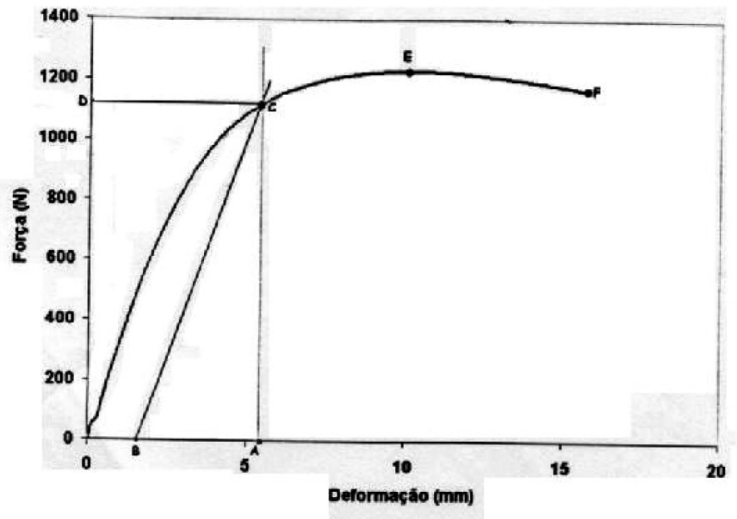

Figura 5

Figura esquemática utilizada para a determinação das propriedades mecânicas do sistema.

Fonte: Laboratório do Centro de caracterização e desenvolvimento de materiais (CCDM) da Universidade Federal de São Carlos - UFSCar - São Paulo (SP), Brasil. (Carga Máxima: força máxima aplicada no corpo de prova durante a realização do ensaio, representada pela letra $E$. Deslocamento máximo: máxima deformação alcançada durante a realização do ensaio. Rigidez compressiva: coeficiente angular do trecho retilíneo da curva força $x$ deformação, representado pelo segmento BC. Carga de escoamento: força necessária para produzir deformação permanente igual a 0,02 vezes o comprimento útil do elemento longitudinal, representada pela letra $D$. Deslocamento em 2\%: deformação permanente igual a 0,02 vezes o comprimento útil do elemento longitudinal, representada pela letra $B$. Desvio de deslocamento $2 \%$ : representado pela letra $A$, definindo o início da deformação plástica. Deslocamento elástico: diferença entre o desvio de deslocamento e deslocamento $2 \%$ ).

Os valores médios dos ensaios de compressão dos grupos 1 e 2 podem ser observados e comparados na tabela 5 .

Com relação à integridade dos componentes metálicos implantáveis dos dois sistemas de parafusos poliaxiais $\left(\right.$ Dytech $^{\circledR}$ e Lock $1^{\circledR}$ ), não foi observado nenhum tipo de escorregamento, quebra e/ou soltura de porcas, parafusos ou qualquer outro componente dos sistemas.

Como não há nenhuma recomendação ou referência estabelecida pela norma ASTM F1717-04 em relação aos valores dos ensaios, a análise refere-se a uma comparação entre valores obtidos.

Aplicado teste $t$ de Student, foram encontrados os seguintes valores de $\mathrm{p}$ :

Desvio 2\%: $\mathrm{p}=0,001$

Deslocamento elástico: $\mathrm{p}=0,001$

Rigidez: $\mathrm{p}=0,001$

Carga de escoamento: $\mathrm{p}=0,45$ (diferença não-significativa)

Carga máxima: $\mathrm{p}=0,15$ (diferença não-significativa)

Deslocamento máximo: $\mathrm{p}=0,001$ 
TABELA 3 - Valores obtidos no ensaio de compressão do Grupo 1

\begin{tabular}{|c|c|c|c|c|c|c|}
\hline Amostra & $\begin{array}{c}\text { Desvio } \\
2 \%(\mathrm{~mm})\end{array}$ & $\begin{array}{l}\text { Deslocamento } \\
\text { elástico }(\mathrm{mm})\end{array}$ & $\begin{array}{l}\text { Rigidez } \\
(\mathrm{N} / \mathrm{mm})\end{array}$ & $\begin{array}{c}\text { Carga } \\
\text { escoamento (N) }\end{array}$ & $\begin{array}{l}\text { Carga máxima } \\
\text { (N) }\end{array}$ & $\begin{array}{l}\text { Deslocamento } \\
\text { máximo }(\mathrm{mm})\end{array}$ \\
\hline & & & 344,78 & 798,51 & 934,81 & \\
\hline \multirow[t]{2}{*}{ CP1 } & 4,42 & 2,9 & $(34,48 \mathrm{Kgf} / \mathrm{mm})$ & $(79,85 \mathrm{Kgf})$ & $(93,48 \mathrm{Kgf})$ & 15,23 \\
\hline & & & 288,62 & 818,47 & 929,99 & \\
\hline \multirow[t]{2}{*}{ CP2 } & 4,99 & 3,47 & $(28,86 \mathrm{Kgf} / \mathrm{mm})$ & $(81,84 \mathrm{Kgf})$ & $(92,99 \mathrm{Kgf})$ & 14,83 \\
\hline & & & 339,67 & 797,14 & 1036,7 & \\
\hline \multirow[t]{2}{*}{ CP3 } & 4,46 & 2,94 & $(33,97 \mathrm{Kgf} / \mathrm{mm})$ & $(79,71 \mathrm{Kgf})$ & $(103,67 \mathrm{Kgf})$ & 15,46 \\
\hline & & & 324,36 & 804,71 & 967,17 & \\
\hline Média & 4,62 & 3,1 & $(32,43 \mathrm{Kgf} / \mathrm{mm})$ & $(80,47$ Kgf) & $(96,71 \mathrm{Kgf})$ & 15,17 \\
\hline Desvio & & & 31,05 & 11,94 & 60,27 & \\
\hline Padrão & 0,32 & 0,32 & $(3,10 \mathrm{Kgf} / \mathrm{mm})$ & $(1,19 \mathrm{Kgf})$ & $(6,02 \mathrm{Kgf})$ & 0,32 \\
\hline
\end{tabular}

Fonte: Universidade Federal de São Carlos - UFSCar - São Carlos (SP), Brasil.

TABELA 4 - Valores obtidos no ensaio de compressão do Grupo 2

\begin{tabular}{lcccccc}
\hline Amostra & $\begin{array}{c}\text { Desvio } \\
\mathbf{2 \%}(\mathbf{m m})\end{array}$ & $\begin{array}{c}\text { Deslocamento } \\
\text { elástico }(\mathbf{m m})\end{array}$ & $\begin{array}{c}\text { Rigidez } \\
(\mathbf{N} / \mathbf{m m})\end{array}$ & $\begin{array}{c}\text { Carga } \\
\text { escoamento }(\mathbf{N})\end{array}$ & $\begin{array}{c}\text { Carga máxima } \\
(\mathbf{N})\end{array}$ & $\begin{array}{c}\text { Deslocamento } \\
\text { máximo }(\mathbf{m m})\end{array}$ \\
\hline CP1 & 1,52 & 6,5 & 142,42 & 892,44 & 908,35 & 6,5 \\
CP2 & 1,52 & 6,05 & 136,26 & 791,43 & 901,43 & 6,05 \\
CP3 & 1,52 & 5,81 & 146,92 & 819,8 & 908,35 & 5,81 \\
Média & 1,52 & 6,12 & 141,87 & 834,56 & 906,04 & 34,46 \\
Desvio padrão & 0 & 0,35 & 5,35 & 52,1 & 4 & 0,35 \\
\hline
\end{tabular}

Fonte: Universidade Federal de São Carlos - UFSCar - São Carlos (SP), Brasil.

TABELA 5 - Valores dos ensaios de compressão dos Grupos 1 e 2

\begin{tabular}{lcccccc}
\hline Grupo & $\begin{array}{c}\text { Desvio } \\
\mathbf{2 \%}(\mathbf{m m})\end{array}$ & $\begin{array}{c}\text { Deslocamento } \\
\text { elástico }(\mathbf{m m})\end{array}$ & $\begin{array}{c}\text { Rigidez } \\
(\mathbf{N} / \mathbf{m m})\end{array}$ & $\begin{array}{c}\text { Carga } \\
\text { escoamento }(\mathrm{N})\end{array}$ & $\begin{array}{c}\text { Carga máxima } \\
(\mathrm{N})\end{array}$ & $\begin{array}{c}\text { Deslocamento } \\
\text { máximo }(\mathbf{m m})\end{array}$ \\
\hline Poliaxiais Dytech 1 ${ }^{\circledR}$ & 4,62 & 3,1 & 324,36 & 804,71 & 967,17 & 15,17 \\
Poliaxiais Lock 2 $^{\circledR}$ & 1,52 & 6,12 & 141,87 & 834,56 & 906,04 & 34,46 \\
\hline
\end{tabular}

Fonte: Universidade Federal de São Carlos - UFSCar - São Carlos (SP), Brasil.

\section{DISCUSSÃO}

A norma ASTM F1717-04 mostrou-se uma ferramenta de grande utilidade para análise das propriedades mecânicas dos sistemas avaliados. Os resultados apresentados mostraram-se consistentes, considerando o grau de repetibilidade dos ensaios ${ }^{12}$.

A fixação com parafuso pedicular da coluna é cada vez mais importante como opção para o cirurgião de coluna e serve para o tratamento de diversas patologias ${ }^{1-3,9}$. É sabido que parafusos poliaxiais costumam ter menor probabilidade de quebra, quando comparados com os monoaxiais ${ }^{8}$ Porém, as diferenças biomecânicas entre parafusos poliaxiais e monoaxiais não são bem conhecidas ${ }^{13}$. Novos designs e técnicas cirúrgicas tem sido constantemente implementadas nas últimas 2 décadas em busca de reduzir a quebra dos parafusos e facilitar suas conexões ${ }^{14,15}$.

Trabalho similar a este foi realizado em 2008, porém os objetivos da comparação do estudo atual são diferentes ${ }^{16}$. Naquele trabalho, foi comparada a resistência das forças de compressão entre os parafusos pediculares poliaxiais e monoaxiais, sendo os últimos mais resistentes. No trabalho atual, comparamos fixação pedicular poliaxial Dytech $^{\circledR}$ com fixação pedicular poliaxial tipo Lock $1^{\circledR}$. Nenhum estudo similar ao nosso, comparando diferentes tipos de fixação pedicular poliaxial foi encontrado.

Estudos biomecânicos observados na literatura utilizam diferentes modelos de montagem. Isso provavelmente se deve ao fato de não existir um modelo experimental aceito universalmente. Shepard ${ }^{10}$, utilizou amostras de coluna de porcos. Góes et al. ${ }^{11}$ utilizaram blocos de madeira. No presente estudo, foram usados blocos de polietileno de peso molecular ultraelevado (UHMWPE), que têm como característica a eliminação dos efeitos de variabilidade das propriedades ósseas e morfométricas ${ }^{12}$.

Os resultados de compressão nas amostras de parafusos poliaxiais com travamento tipo Dytech ${ }^{\circledR}$ tiveram uma carga máxima média de 967,17 N e carga de escoamento média de $804,71 \mathrm{~N}$. Nas amostras dos parafusos poliaxiais 
com travamento tipo Lock $1^{\circledR}$ tivemos uma carga máxima média de 906,04 $\mathrm{N}$ e carga de escoamento média de 834,56 N. Como já ressaltado, não encontramos trabalho similar na literatura como objeto de comparação.

Todos os ensaios deste trabalho incluíram o uso do estabilizador transversal. Defino et al. não encontraram estabilidade adicional referente ao estabilizador transversal ${ }^{3}$. Góes et al. mostraram que a utilização do estabilizador transversal aumenta significativamente a estabilidade mecânica do sistema no plano frontal e, principalmente, no plano rotacional ${ }^{11}$.

\section{CONCLUSÃO}

No presente estudo, a montagem com parafusos polioaxiais com travamento tipo Dytech ${ }^{\circledR}$ mostrou valores de rigidez e carga máxima maiores, quando comparada com montagens com parafusos poliaxiais com travamento tipo Lock $1^{\circledR}$. Somente os valores de rigidez apresentaram significância estatística. Já o sistema de parafusos com travamento Lock $1^{\circledR}$ apresentou carga de escoamento e deslocamento máximo maiores que a montagem com parafusos poliaxiais com travamento Dytech ${ }^{\circledR}$. Porém, a significância estatística foi observada somente em relação ao deslocamento máximo.

\section{REFERÊNCIAS}

1. Leite VC, Okubo R, Shimano AC, Kandziora F. Influência da profundidade da inserção do parafuso no pedículo vertebral sobre o torque de inserção e a resistência ao arrancamento. Coluna/Columna. 2008;7(2):128-32.

2. Suk SI, Kim WJ, Lee SM, Kim JH, Chung ER. Thoracic pedicle screw fixation in spinal deformities: are they really safe?. Spine (Phila $\mathrm{Pa}$ 1976). 2001;26(18):2049-57.

3. Defino HLA, Moro CA, Fuentes AER, Paulin JB. Avaliação da angulação dos parafusos e utilização do estabilizador transversal na estabilidade do sistema de fixação pedicular. Rev Bras Ortop. 1996;31(11): 925-30.

4. Boucher HH. A method of spinal fusion. J Bone Joint Surg Br. 1959;41:248-59.

5. Roy-Camile R, Saillant G, Marzel C. Internal fixation of the lumbar spine with pedicle screw plating. Clin Orthop Relat Res.1986;(203):7-17.

6. Roy-Camile R, Saillant G, Marzel C. Plating of thoracic, thoracolumbar, and lumbar injuries with pedicle screw plates. Clin Orthop North Am. 1986;17(1):147-59.

7. Krag MH. Overview of the options and posterior internal fixation devices. In: Frymoyer JW.The adult spine: Principles and practice. New York: Raven Press; 1991. p. 1919-45.
8. Fogel GR, Reitman CA, Liu W, Esses SI. Physical characteristics of polyaxial-headed pedicle screws and biomechanical comparison of load with their failure. Spine (Phila $\mathrm{Pa}$ 1976). 2003;28(5):470-3.

9. Suk SI, Lee SM, Chung ER, Kim JH, Kim SS. Selective thoracic fusion with segmental pedicle screw fixation in the treatment of thoracic idiopathic scoliosis: more than 5-year follow-up. Spine (Phila Pa 1976). 2005;30(14):1602-9.

10.Shepard MF, Davies MR, Abayan A, Kabo JM, Wang JC. Effects of polyaxial pedicle screws on lumbar construct rigidity. J Spinal Disord Tech. 2002;15(3):233-6.

11. Góes LA, Shimano CA, Defino LAH. Estudo biomecânico da participação do estabilizador transvesal na estabilidade mecânica das fixações vertebrais com o fixador interno. Coluna/Columna. 2004;3(3):122-7.

12.ASTM F1717-04. Standard Test Methods for Spinal Implants Constructs in a Vertebrectomy Model. ASTM International: Medical Devices, Emergency Medical Services. Book of Standards. v. 13.01; 2008.

13.Cunningham BW, Sefter JC, Shono Y, McAfee PC. Static and cyclical biomechanical analysis of pedicle screw spinal constructs. Spine (Phila Pa 1976). 1993;18(12):1677-88.
14.McKinley TO, McLain RF, Yerby SA, Sharkey NA, Sarigul-Klijn N, Smith TS. Characteristics of pedicle screw loading. Effect of surgical technique on intravertebral and intrapedicular bending moments. Spine (Phila $\mathrm{Pa}$ 1976). 1999;24(1):18-24.

15.Stanford RE, Loefler AH, Stanford PM, Walsh WR. Multiaxial pedicle screw designs: static and dynamic mechanical testing. Spine (Phila Pa 1976). 2004;29(4):367-75.

16.França LC, Oliveira MPC, Falcon RS, Porto Filho MA, Rugani MG, Werlang PM, et al. Estudo biomecânico comparativo da resistência às forças de compressão entre os parafusos pediculares poliaxiais e monoaxiais. Coluna/Columna. 2008;7(2):133-8.

\author{
Correspondência \\ Mauricio Pagy de Calais Oliveira \\ Rua Paracatu, 878/801 \\ CEP: 30180-090 - Belo Horizonte \\ (MG), Brasil. \\ Tel: (31) 3295-6291 \\ E-mail: mauriciocalais@hotmail.com
}

